\title{
TRATAMIENTO QUIRÚRGICO DE LA INCONTINENCIA URINARIA DE ESFUERZO EN NUESTRO MEDIO: ANÁLISIS DE RESULTADOS
}

\author{
E. REDONDO GONZÁLEZ, M. LUJÁN GALÁN, A. PÁEZ BORDA, \\ J.M. GÓMEZ DE VICENTE, G.M. ESPINALES CASTRO, A. BERENGUER SÁNCHEZ
}

Servicio de Urología. Hospital Universitario de Getafe. Madrid.

Actas Urol Esp. 27 (9): 700-706, 2003

\section{RESUMEN}

TRATAMIENTO QUIRÚRGICO DE LA INCONTINENCIA URINARIA DE ESFUERZO EN NUESTRO MEDIO: ANÁLISIS DE RESULTADOS INTRODUCCIÓN: La corrección quirúrgica es actualmente el método más efectivo de tratamiento para la incontinencia urinaria de esfuerzo (IUE) de entre las diferentes opciones terapéuticas de las que se dispone en la actualidad. El objetivo de este trabajo consiste en comparar la eficacia de los distintos grupos de técnicas empleadas para el tratamiento de la IUE en nuestras pacientes.

MATERIAL Y MÉTODO: Hemos llevado a cabo un análisis retrospectivo de las pacientes intervenidas en nuestro Servicio por IUE durante el periodo comprendido entre abril de 1991 y julio de 1999 (213 intervenciones en 194 pacientes), agrupando los procedimientos en 3 grupos: técnicas abdominales, técnicas abdomino-vaginales, y técnicas de cabestrillo. La tasa de éxito de la intervención fue valorada mediante tres categorías: continencia total, mejoría clínicamente significativa, y ausencia de mejoría. La comparación bivariante de proporciones se realizó mediante la prueba exacta de Fisher y el test de Chi-cuadrado. La asociación de variables cualitativas se evaluó mediante el test de Chi-cuadrado: se utilizó un modelo de Cox para el análisis multivariante de los factores predictores de continencia, y curvas de Kaplan-Meier para la evaluación de la supervivencia de la continencia post-operatoria. Para todos ellos se tomó como nivel de significación estadística una $\mathrm{p}<0,05$.

RESULTADOS: La tasa de continencia total de nuestra serie fue del 54,5\% (116 pacientes continentes) a los 2 años. En el grupo de mejoria clínica significativa fueron incluidas 33 pacientes (15,5\%) y en el grupo de pacientes sin mejoria 64 pacientes (30\%) (Figs. 3 y 4). Las complicaciones más frecuentes fueron la presencia de dolor suprapúbico (33\%), la infección de la herida (20\%), la retención de orina post-operatoria (26\%), y la presencia de residuo post-miccional significativo (24\%). Ninguno de los múltiples factores analizados, se mostraron como factores predictivos independientes para la continencia post-operatoria. En el análisis actuarial, tras la comparación de las curvas de Kaplan-Meier correspondientes a cada técnica, objetivamos que no existen diferencias entre las distintas técnicas (logrank; $\mathrm{p}=0,41$ ) presentando la técnica de sling un mayor número de complicaciones.

CONCLUSIONES: La tasa de continencia total post-quirúrgica de nuestra serie es de un $54,5 \%$ a los dos años, encontrando un total de $70 \%$ de respuestas clínicamente satisfactorias. No parece existir una técnica claramente superior a las demás en cuanto a eficacia para nuestras pacientes, presentando la técnica de sling un porcentaje superior de complicaciones. No hemos encontrado factores pre o post-operatorios que influyan de modo independiente en la predicción de la continencia post-quirúrgica.

PALABRAS CLAVE: Incontinencia urinaria de stress. Tratamiento quirúrgico. Resultados.

\section{ABSTRACT}

OUTCOME ANALYSIS FOR SURGICAL TREATMENT OF STRESS URINARY INCONTINENCE IN OUR SETTING

INTRODUCTION AND OBJECTIVE: Surgical repair is the most effective option for the treatment of stress urinary incontinence (SUI) between the different therapeutical options availables at present. The main objective of our study is to compair the outcome of the different techniques employed in the treatment of SUI in our setting.

METHOD: We have performed a retrospective analysis of the patients who underwent surgical intervention for SUI between 1991 to 1999 (213 surgical interventions in 194 patients) clustering the surgical procedures into three groups: abdominal, abdomino-vaginal, and sling procedures. The results of the treatment were defined as follows: total continence, significant improvement and insufficient improvement. Comparison of continence rates was performed with $\chi^{2}$ test and Fisher's exact test. Association between qualitative variables was also evaluated by means of $\chi^{2}$ test. Multivariate analysis of predictive factors was performed with a Cox model. The outcome was also evaluated by Kaplan-Meier's curves, and comparisons made with log-rank test. Statistical significance level was established for $\mathrm{p}<0.05$.

RESULTS: Global cure rate at 24 months was 54.5\% (116 patients). Significant improvement was observed in 33 patients (15.5\%), and insufficient improvement was seen in 64 patients (30\%). The most frequent postoperative complications were suprapubic pain (33\%), acute urinary retention $(26 \%)$, significant postvoiding residual urine $(24 \%)$ and wound seroma or infection $(20 \%)$. None of the analyzed factors (age, weight, number of births, preoperative pads, postoperative acute urinary retention, and need for postoperative bladder clean intermitent catheterization were independient predictive factors for postoperative continence. The actuarial analysis with Kaplan-Meier curves shows no statistical differences between the studied techniques (log rank $\mathrm{p}=0.41)$. Sling techniques presented with a superior rate of most postoperative complications.

CONCLUSIONS: The cure rate of our serie was $54.5 \%$ at 24 months, with a $70 \%$ of clinically satisfactory responses. With regard to continence status, it seems that there is not a better surgical technique in our hands, presenting sling techniques with a higher rate of postoperative complications. We could not find no pre o postoperative independient factors as predictors of postoperative continence. 
$\mathrm{L}$ a incontinencia urinaria de stress (IUE) en la mujer es un problema relativamente frecuente. En un estudio clásico realizado sobre mujeres que acudieron a las consultas de medicina general, se objetivó que hasta un 57\% de las mujeres entre 45 y 64 años presentaban incontinencia urinaria de esfuerzo de suficiente cuantía como para representar para ellas un problema higiénico o social $^{1}$. Estudios más recientes ${ }^{2-3}$ siguen cifrando este problema en porcentajes de un 52$61 \%$ de mujeres de más de 20 años de edad.

Si bien las opciones terapéuticas a este problema son múltiples, la corrección quirúrgica de la IUE es actualmente el método más efectivo de tratamiento de las que se dispone en la actualidad. La literatura está repleta de técnicas quirúrgicas utilizadas a lo largo del tiempo para tratar este problema, muchas de las cuales han sido ya abandonadas por la mayoría de los grupos en la actualidad por haberse demostrado ineficaces.

Actualmente, basándonos en los fundamentos comunes de tratamiento, existen cuatro grandes grupos de intervenciones para el tratamiento de la IUE: intervenciones por vía vaginal, intervenciones por vía abdominal, intervenciones por vía abomino-vaginal o combinadas, y técnicas de cabestrillo. Otras técnicas como las inyecciones periuretrales o el esfínter urinario artificial son reservadas para casos muy seleccionados y en los que han fracasado otras técnicas.

El objetivo de este trabajo consiste en comparar la eficacia de los distintos grupos de técnicas en nuestras manos para el tratamiento de la IUE.

\section{MATERIAL Y MÉTODO}

Hemos llevado a cabo un análisis retrospectivo de las pacientes intervenidas en nuestro Servicio por IUE durante el periodo comprendido entre abril de 1991 (comienzo de la actividad quirúrgica del Servicio de Urología de este Hospital) y julio de 1999 (introducción de las técnicas laparoscópicas como primera opción de tratamiento quirúrgico de la IUE). En este periodo de tiempo fueron realizadas en nuestro servicio 213 intervenciones quirúrgicas en 194 pacientes afectas de IUE (1,09 intervenciones por paciente).

Para un mejor análisis de los datos, las diferentes intervenciones empleadas fueron agrupadas en 3 los tres grupos de técnicas que solemos llevar a cabo (no realizamos intervenciones por vía vaginal): técnicas abdominales (Burch $\mathrm{y}$ Marshall-Marchetti), técnicas abomino-vaginales o técnicas combinadas (Raz I, Raz II y Leach) y técnicas de cabestrillo (cabestrillo con parche vaginal y con fascia de recto abdominalis).

La tasa de éxito de la intervención fue evaluada tres categorías: paciente continente (aquella que a la anamnesis refiere continencia total, no precisando de ninguna compresa para mantenerse seca); paciente con mejoría clínica significativa (aquella paciente que a la anamnesis refiere persistencia de una incontinencia menor que previamente a la intervención, pero con resultado subjetivo satisfactorio); y paciente sin mejoría (aquella que ha experimentado una mejoría clínica leve, nula, o insuficiente, y con resultado subjetivo insatisfactorio). Para el análisis de supervivencia quirúrgico, las categorías de mejoría clínica significativa e incontinentes, fueron agrupadas como pacientes incontinentes.

El tiempo de seguimiento fue evaluado por separado en las pacientes continentes e incontinentes, de tal modo que en las pacientes continentes se analizó el tiempo de seguimiento (número de meses de seguimiento/número de pacientes continentes) y en las pacientes incontinentes (mejorías y no mejorías) se analizó el tiempo de continencia (número de meses de continencia/número de pacientes incontinentes), puesto que a partir del momento en que aparece la IUE de nuevo en la paciente no resulta informativo el posterior seguimiento.

Se emplearon la prueba exacta de Fisher y el test de Chi-cuadrado para la comparación bivariante de proporciones. La asociación de variables cualitativas se analizó mediante el test de Chi-cuadrado. El análisis multivariante de los factores predictores de continencia, se llevó a cabo mediante un modelo de Cox. La evaluación de la continencia post-operatoria se analizó mediante curvas de Kaplan-Meier, y la comparación de las curvas mediante el test de log-rank. Todos los intervalos de confianza y el nivel de significación estadística se calcularon para una $\mathrm{p}<0,05$.

El tratamiento estadístico de los datos fue llevado a cabo con soporte informático, mediante el programa SPSS-8 para Windows. 


\section{RESULTADOS}

Estadística descriptiva de la serie

La edad de las pacientes intervenidas osciló entre 27 y 81 años (media de 53,9 $\pm 3,1$ años), mientras que la talla media fue de $154,45 \pm 4,27$ $\mathrm{cm}$, y un peso medio de $70,41 \pm 2,9 \mathrm{Kg}$ (rango 52 $105 \mathrm{Kg}$ ). De las 194 pacientes intervenidas, 15 $(7,73 \%)$ tenían antecedentes de histerectomía previa, y $24(12,37 \%)$ habían sido sometidas previamente a uretrocervicopexia. El número medio de partos de las pacientes intervenidas fue de 3,13 (rango 0-8), y el número medio de compresas que las pacientes utilizaban preoperatoriamente fue de $4,15 \pm 0,2$ compresas (1-10). 36 pacientes $(18,56 \%)$ presentaban además de IUE clínica de urgencia miccional y $15(7,73 \%)$ incontinencia por urgencia. 35 pacientes presentaron a la exploración un cistocele grado 0 (18\%), 96 un cistocele grado I $(49,5 \%), 42$ un cistocele grado II (21,6\%), 7 un cistocele grado III $(3,6 \%)$, y 14 un cistocele grado IV $(7,3 \%)$.

La distribución de técnicas empleadas en nuestra muestra, fue: 40 intervenciones por vía abdominal $(18, \%), 141$ intervenciones por vía vaginal $(66,2 \%)$, y 32 cirugías de sling vaginal (15\%) (Fig. 1).

\section{Resultados globales de la serie}

La tasa de éxito de la serie, considerando como éxito la continencia total de la paciente evaluada mediante anamnesis fue del 54,5\% (116 pacientes continentes). En el grupo de mejoría

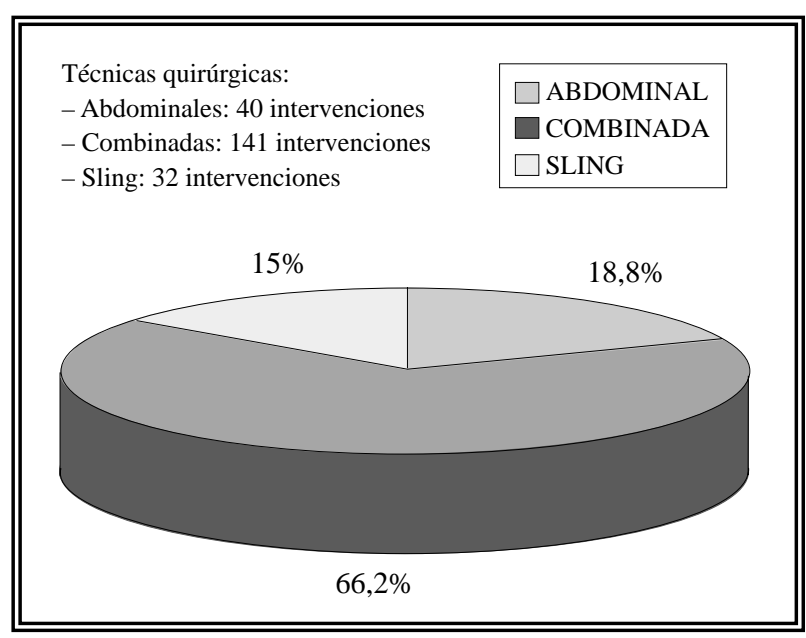

FIGURA 1. Proporciones comparativas de los diferentes grupos de intervenciones de IUE. clínica significativa fueron incluidas 33 pacientes $(15,5 \%)$ y en el grupo de pacientes sin mejoría 64 pacientes (30\%) (Fig. 2).

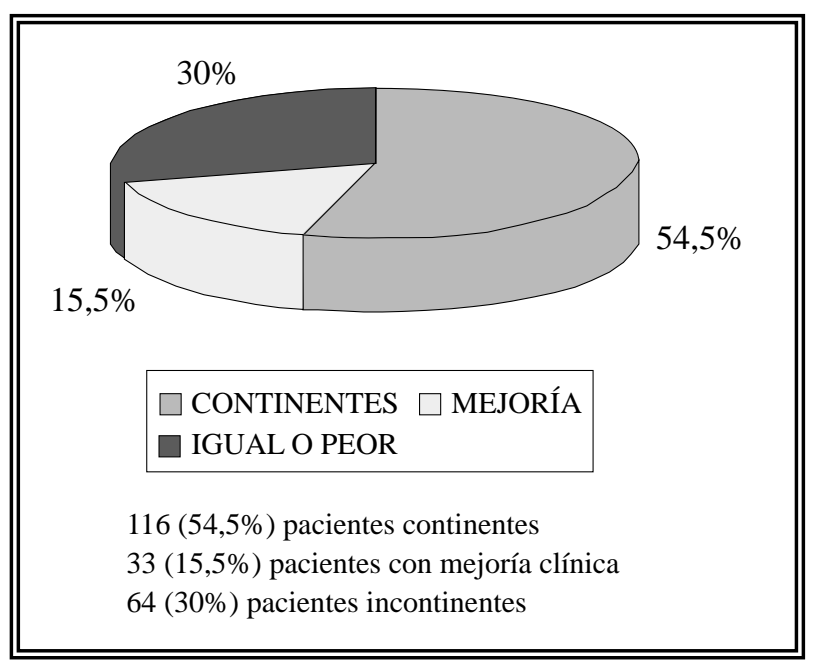

FIGURA 2. Resultados globales en cuanto a la continencia total.

En las pacientes continentes, el tiempo medio de seguimiento fue de 25,76 $\pm 2,54$ meses (rango 1-116 meses). En las pacientes incontinentes (mejorías significativas y no mejorías), el tiempo medio hasta la aparición de incontinencia fue de $8,52 \pm 0,87$ meses (de inmediata - 41 meses). El número medio de compresas utilizadas post-operatoriamente fue de 1,25 $\pm 0,19$ (0-10 compresas).

Las complicaciones más frecuentes fueron la presencia de dolor suprapúbico en 72 pacientes (33\%), la infección de la herida quirúrgica en 42 pacientes (20\%), la retención de orina post-operatoria en 56 pacientes (26\%), y la presencia de residuo post-miccional significativo en 52 pacientes (24\%).

En el estudio multivariante, los factores: edad, peso, talla, no de compresas previo, no de partos, intervenciones quirúrgicas previas, cirugias previas de IUE, retención aguda de orina post-quirúrgica, o necesidad de cateterismos vesicales intermitentes post-operatorios, no demostraron ser factores predictivos independientes para la continencia.

\section{Resultados comparativos de las diferentes técnicas}

Para realizar el análisis comparativo entre las diferentes técnicas, hemos considerado a las pacientes tratadas en dos grupos, pacientes con- 
tinentes y pacientes incontinentes. Con esta premisa, los resultados comparativos se ven reflejados en la Tabla I, donde se objetiva que la técnica de sling proporciona una mayor tasa de continencia (75\%) frente a las técnicas abdominales y vaginales (60\% y $48 \%$ respectivamente), siendo esta diferencia estadísticamente significativa $(p<0,05)$. Sin embargo, cuando realizamos un análisis actuarial (Fig. 3), tras la comparación de las curvas de Kaplan-Meier correspondientes a cada técnica, objetivamos que no existen diferencias estadísticamente significativas entre las distintas técnicas (log-rank; $\mathrm{p}=0,41$ ).

En cuanto a las complicaciones post-quirúrgicas tanto la presencia de dolor importante postoperatorio, como la aparición de RAO, como el residuo post-miccional significativo, fueron significativamente mayores con la cirugía de sling $(\mathrm{p}<0,05)$. En cuanto a la infección de la herida quirúrgica, pese a ser más frecuente en el sling (34\% frente a 20 y $16 \%$ de las técnicas abdominal y vaginal respectivamente) la diferencia no llegó a alcanzar el nivel de significación estadística $(\mathrm{p}=0,068)$ (Tabla II).

\section{TABLA I}

TABLA DE RESULTADOS COMPARATIVOS ENTRE LOS DISTINTOS TIPOS DE INTERVENCIÓN PARA LA IUE

\begin{tabular}{||l|c|c|c||}
\hline \multicolumn{4}{|c|}{ Resultados comparativos } \\
\hline & Continentes & Incontinentes & Total \\
\hline Abdominal & $24(60 \%)$ & $16(40 \%)$ & 40 \\
\hline Combinada & $68(48 \%)$ & $73(52 \%)$ & 141 \\
\hline Sling & $24(75 \%)$ & $8(25 \%)$ & 32 \\
\hline Total & 116 & 97 & 213 \\
\hline
\end{tabular}

$(\mathrm{p}=0,017)$

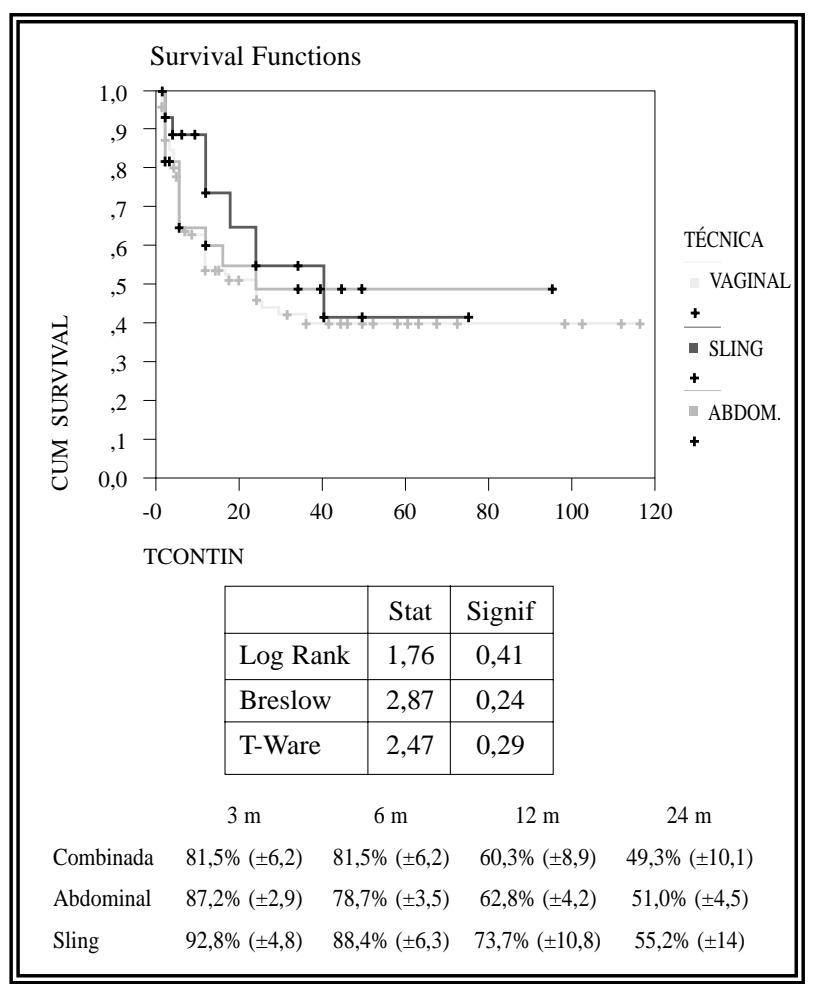

FIGURA 3. Curvas de Kaplan-Meier de supervivencia para los distintos tipos de intervención.

\section{DISCUSIÓN}

La comparación de resultados en el tratamiento quirúrgico de la IUE resulta muy dificultosa por varios motivos: la falta de uniformidad de criterio para la selección de las pacientes, la falta de cuantificación objetiva de la importancia de la incontinencia, la falta de uniformidad en la nomenclatura a la hora de asignar nombre al procedimiento realizado, la falta de uniformidad para describir los éxitos y fracasos del tratamiento, y la falta de uniformidad a la hora de compa-

TABLA II

FRECUENCIA DE LAS COMPLICACIONES MÁS FRECUENTEMENTE DETECTADAS SEPARADAS SEGÚN EL TIPO DE INTERVENCIÓN REALIZADA

\begin{tabular}{|c|c|c|c|c|c|}
\hline \multicolumn{6}{|c|}{ Complicaciones post-quirúrgicas } \\
\hline & $\begin{array}{c}\text { Total } \\
\mathrm{n}\end{array}$ & $\begin{array}{c}\text { Abdominales } \\
\text { n (\%) }\end{array}$ & $\begin{array}{c}\text { Combinadas } \\
\text { n (\%) }\end{array}$ & $\begin{array}{l}\text { Sling } \\
\text { n (\%) }\end{array}$ & $\mathrm{p}$ \\
\hline Dolor post-quirúrgico & 72 & $6(15 \%)$ & $52(36,9 \%)$ & $14(43,7 \%)$ & 0,016 \\
\hline RAO & 56 & $2(5 \%)$ & $38(27 \%)$ & $16(50 \%)$ & $<0,001$ \\
\hline Residuo & 52 & $4(10 \%)$ & $36(25,6 \%)$ & $12(37,5 \%)$ & 0,023 \\
\hline Seroma/Infección & 42 & $8(20 \%)$ & $23(16,4 \%)$ & $11(34,4 \%)$ & 0,068 \\
\hline
\end{tabular}


rar series. Para tratar de evitar en parte estos problemas, en nuestra serie hemos utilizado varios tipos de estrategias.

Para tratar de cuantificar de un modo objetivo la importancia de la incontinencia se han empleado distintos métodos y clasificaciones clíni$\operatorname{cas}^{4,5}$. No obstante, ninguna de ellas es de uso generalizado. Nosotros hemos optado por recoger el número de compresas que utiliza la paciente antes y después de intervenirse como método de cuantificación de la incontinencia, que si bien puede considerarse muy subjetivo para la comparación entre pacientes, si permite contrastar de un modo muy fiable a cada paciente consigo misma antes y después de la intervención.

Debido a la falta de uniformidad a la hora de nominar los procedimientos quirúrgicos realizados, y a que en muchos casos, cada cirujano introduce sus propias variantes personales, parece lógico agrupar las diferentes intervenciones en grandes grupos con fundamentos técnicos comunes para el análisis comparativo, realizado la comparación de técnicas abdominales, frente a técnicas vaginales y frente a técnicas de sling.

Otro problema importante a la hora de comparar distintas series, es que en muchas de ellas se habla de "éxito" y "fracaso" quirúrgico, refiriéndose a diferentes objetivos terapéuticos. Así para algunos autores, el éxito es la continencia total, mientras que para otros, el éxito se trata de la mejoría de la paciente, e incluso en algunos trabajos, no llega a haber una definición clara de estos términos. Debido a la escasa popularidad del empleo de los cuestionarios de incontinencia urinaria $^{6}$ y calidad de vida $(\mathrm{QoL})^{7}$ y a su reciente validación al castellano ${ }^{8,9}$, la mayoría de las pacientes de nuestra serie han sido sometidas a intervención antes de su empleo estandarizado, por lo que para este análisis nos hemos basado en los 3 items descritos previamente. No obstante, y como objetivo para posteriores análisis, el empleo de cuestionarios validados de incontinencia urinaria y calidad de vida pre y post-operatorios parece el mejor método para comparar distintas series.

La comparación de series con distinto tiempo de seguimiento puede llevar a equívocos en el análisis del éxito o del fracaso de la intervención. El único método estadístico que permite obviar esta dificultad, es el análisis actuarial de los datos mediante curvas de supervivencia como son las curvas de Kaplan-Meier. Debido al uso generalizado de las técnicas combinadas a mediados de los años 90 por la mayoría de gru$\operatorname{pos}^{10}$, y a la popularización más tardía de la técnica de sling para el tratamiento de las incontinencias tipo I y $\mathrm{II}^{11}$, la mayoría de las pacientes intervenidas mediante este tipo de técnicas, lo han sido más recientemente, y por tanto presentan un menor tiempo de seguimiento. De hecho, si realizamos un análisis de los datos basado en la tasa global de éxitos, pudiera parecer que la técnica de sling es significativamente más eficaz que el resto (Tabla I). El análisis actuarial no confirma estos datos, sino que indica que el éxito de este tipo de cirugía parece cimentarse en los primeros meses de seguimiento, pero su eficacia decae a lo largo del tiempo hasta hacerse a los 2 años prácticamente similar al resto (Fig. 3).

Finalmente, somos conscientes de que existe un sesgo de selección implícito a nuestra serie (del mismo modo que en todas las series retrospectivas), y que hace difícilmente comparable los distintos grupos de pacientes, puesto que en los casos más severos, en las disfunciones esfinterianas intrínsecas, y en los casos recidivados se han empleado técnicas que se suponen más definitivas (técnica de sling fundamentalmente) que en los casos primarios, y es quizá debido a ello, que la técnica de sling no se demuestra como más eficaz que la demás.

El global de datos de nuestra serie demuestra ser bastante homogéneo con la mayoría de grupos publicados en cuanto a la edad, número de compresas preoperatorias, urgencia miccional clínica, y tendencia a la obesidad de las pacientes $^{12-14}$. Las curvas de Kaplan-Meier, evidencian, como ya han señalado otros autores ${ }^{15}$, que el resultado de la intervención va disminuyendo progresivamente a lo largo del seguimiento, y que parece estabilizarse a los 24 meses $^{16}$, si bien esta última afirmación no puede extraerse con seguridad de nuestra serie, pues es alrededor de esa cifra donde se obtiene la mediana de seguimiento. El tiempo medio de continencia en las pacientes que desarrollan recidiva de la IUE, se cifra en los 8 meses, cifra muy similar a otras series que incluyen este dato dentro de su análisis ${ }^{16,17}$. 
Clásicamente a las técnicas abdominales se le atribuyen por diversos autores un porcentaje de éxito entre el 80 y el 100\% de los $\operatorname{casos}^{13,18,19}$. En el meta-análisis publicado por la $\mathrm{AUA}^{20}$, las series con seguimiento medio entre 12 y 23 meses obtuvieron entre un 78 y un $91 \%$ de buenos resultados, entendiendo como tal las curaciones y las mejorías significativas. Sin embargo, algunas series han publicado porcentajes de éxito similares al nuestro ${ }^{15,21}$, y claramente inferiores a los publicados por los otros grupos. Los resultados obtenidos con las técnicas abdominovaginales son variables y en muchos casos sensiblemente inferiores al resto de las técnicas ${ }^{22-25}$, oscilando según la reciente revisión de la AUA entre el 56 y el $92 \%$, y con una media del $67 \%{ }^{20}$. En nuestro grupo de pacientes, el porcentaje de éxitos es inferior al resto, sin llegar a alcanzar este dato un nivel de significación estadística en el análisis de supervivencia, del mismo modo que se observó en publicaciones previas de nuestro grupo $^{26,27}$. En cuanto a los resultados obtenidos con las técnicas de sling, también existe mucha variación entre los resultados que apuntan los diferentes estudios ${ }^{15,28-30}$ y que oscilan entre un $44 \%^{31}$ y un $98 \%{ }^{32}$. En nuestra serie, las técnicas de sling no se muestran como superiores a los demás tipos de técnicas cuando se realiza un análisis actuarial de las técnicas, lo que puede estar justificado en parte debido al sesgo de selección de pacientes al que nos hemos referido previamente, y que ya ha sido apuntado por otros autores, en estudios similares al nuestro ${ }^{21}$.

\section{CONCLUSIONES}

La tasa de continencia total post-quirúrgica de nuestra serie es de un $54,5 \%$ a los dos años. A este mismo tiempo, un $70 \%$ de las pacientes se encuentran significativamente mejor que antes de intervenirse. En nuestra serie, no parece existir un grupo de técnicas claramente superior al resto en cuanto a eficacia se refiere. No hemos encontrado factores pre o post-operatorios que influyan de modo independiente en la predicción de la continencia post-quirúrgica. Será conveniente la realización de un estudio prospectivo sobre calidad de vida pre y post-operatorio en las pacientes sometidas a intervención quirúrgica por IUE para determinar si la mejoría se puede considerar como un éxito o un fracaso de la intervención.

\section{REFERENCIAS}

1. DIOKNO A, BROCK B.: Prevalence of urinary incontinence and other urological symptoms in the non institutionalized elderly. J Urol 1986; 136 (5): 1022 .

2. BORTOLOTTI A, BERNARDINI B, COLLI E, DIBENEDETTO P, NACCI GG, LANDONI $M$ et al.: Prevalence and risk factors for urinary incontinence in Italy. Eur Urol 2000; 37: 30.

3. HANNENSTEAD YS, RORTVEIT G, SANDVIK $H$, HNSKAAR SA.: A community-based epidemiological survey of female urinary incontinence: The Norwegian Epincont study. J Clin Epidemiol 2000; 53: 1150.

4. OBRNICK A, BUNNE G, ULMSTEN U, INGELMANSUNDBERG A.: Urethral pressure profile before, during and after pubococcygeal repair of stress incontinence. Acta Obstet Gynecol Scand 1978; 47: 49.

5. MOURISTSEN L, BERILD G, HERTZ J.: Comparison of different methods of quantification of urinary leakage in incontinent women. Neurourol Urodyn 1989; 8: 579.

6. BLACK NA, GRIFFITHS JM, POPE C, STANLEY J, BROWLING A, ABEL PD.: Development of a symptom severity index and a symptom impact index of stress incontinence in woman. Neurol Urodynamics 1996: 630.

7. RICHARD F, HAAB F, AMARENCO G, BALLANGER P.: Development and validation of a new specific questionnaire to assess women's health related quality of life (HRQL) in urinary incontinence. Eur Urol 1999: 35 (suppl 2): 71.

8. VILA MA, FERNÁNDEZ MJ, FLORENSA E, OREJAS V, BAQUE A, ROMEA S.: Validación de un cuestionario para el diagnóstico de incontinencia urinaria. Aten Primaria 1997; 19 (3): 121.

9. BADIA LLACH X, CASTRO DÍAZ D, PERALES CABAÑAS L, PEÑA OUTERIÑO JM, MARTÍNEZ AGULlÓ E, CONEJERO SUGRAÑES J, ARAÑó BELTRÁN P, MARQUÉS QUEIMADELOS A, ROSET GAMISANS M, PERUELO ESCOBAR N.: Elaboración pre-eliminar del cuestionario para la clasificación clínica de la incontinencia urinaria IU-4. Actas Urol Esp 1999; 23 (7): 576.

10. GEE WF, HOLTGREWE HL, ALBERTSEN PC, LITWIN MS, MANYAK MJ, O'LEARY MP, PAINTER MR, BLIZZARD RT, FENNINGER RB, EMMONS L.: Practice trends of American urologist in the treatment of impotence, incontinence, and infertility. $J$ Urol 1996; 156: 1778.

11. KIM HL, GERBER GS, PATEL RV, HOLLOWELL CM, BALES GT.: Practice patterns in the treatment of female urinary incontinence: a postal an internet survey. Urology 2001; 57: 45.

12. LITWILLER SE, NELSON RS, FONE PD, KIM KB, STONE AR.: Vaginal wall sling: long-term outcome analysis of factors contributing to patient satisfaction and surgical success. J Urol 1997; 157: 1279.

13. RAZ S, SUSSMAN M, ERICKSON DB, BREGG KC, NITTI VW.: The Raz bladder neck suspension: results in 206 patients. J Urol 1992; 148: 845. 
14. MADRID GARCÍA FJ, RIVAS ESCUDERO JA, MADROÑERO CUEVAS C, GARCÍA ALONSO J.: Cabestrillo con pared vaginal en el tratamiento de la incontinencia urinaria de esfuerzo femenina. Análisis de 27 casos. Arch Esp Urol 2001; 54 (3): 247.

15. CLEMENS JQ, STERN JA, BUSHMAN WA, SCHAEFFER AJ.: Long term results of the Stamey bladder neck suspension: direct comparision with the Marshall-Marchetti-Krantz procedure. J Urol 1998; 160: 372 .

16. GUTIÉRREZ BAÑOS JL, DE DIEGO RODRÍGUEZ E, PORTILLO MARTÍN JA, MARTÍN GARCÍA B, HERNÁNDEZ RODRÍGUEZ R, CORREAS GÓMEZ MA, DEL VALLE SCHAAN JI, ROCA EDREIRA A, RADO VELÁZQUEZ MA.: Las técnicas de Raz en el tratamiento de la incontinencia urinaria de esfuerzo en la mujer. Actas Urol Esp 1999; 23 (5): 417.

17. PEREYRA AJ, LEBHERZ TB.: Combined urethrovesical suspension and vaginourethroplasty for correction of urinary incontinence. Obstet Gynecol 1967; 30: 537.

18. HOANG-BOEHM J, KRANSTSCHICK A, HAITSMAN V, ALKEN P.: Burch against Stamey: the truth about posoperative continence rates in long term follow up. J Urol 1998; Suppl abstract 165: 45.

19. JONGEN VH, BROUWER WK.: Comparison of the modified Pereyra procedure using permanent suture material and Burch urethropexy. Eur $J$ Obstet Gynecol Reprod Biol 1999; 84: 7.

20. The surgical management of female stress incontinence. American Urological Association Inc. Clinical Practice Guidelines, 1997.

21. VICENTE PRADOS FJ, ESPEJO MALDONADO M, TALLADA BUÑUEL M, CÓZAR OLMO JM, MARTÍNEZ MORCILLO A, PEDRAJAS DE TORRES G, BUITRAGO SIVIANES S, RODRIGUEZ HERRERA F.: Técnica de Burch en el tratamiento quirúrgico de la incontinencia urinaria de esfuerzo femenina: valoración de resultados y análisis de la causa de los fracasos.

22. EKABIR JJ, MEE AD.: Long-term evaluation of the Gittes procedure for urinary stress incontinence. $J$ Urol 1998; 159: 1208.

23. TEBYANI N, PATEL H, YAMAGUCHI R, ABOSEIF SR.: Percutaneous needle bladder neck suspension for the treatment of stress urinary incontinence in women: long term results. J Urol 2000; 163: 1510.
24. NIGAM AK, OTITE U, BADENOCH DF.: Endoscopic bladder neck suspension revisited. Long term results of Stamey and Gittes procedures. Eur Urol 2000; 38: 677 .

25. O'SULLIVAN DC, CHILTON CP, MUNSON KW.: Should Stamey colposuspension be our primary surgery for stress incontinence. Br J Urol 1995; 75: 457.

26. LUJAN GALÁN M, PÁEZ BORDA A, BUSTAMANTE ALARMA S, MARTÍN OSES E, SÁNCHEZ SÁNCHEZ E, ZÁRATE RODRÍGUEZ E, LLANES GONZÁLEZ L, LLORENTE ABARCA C, BERENGUER SÁNCHEZ A.: Análisis de los resultados tras la cirugía de la incontinencia urinaria de stress en la mujer. Urod A 1996; 127.

27. LUJAN GALÁN M, PÁEZ BORDA A, BUSTAMANTE ALARMA S, FERNÁNDEZ GONZÁLEZ I, GAGO JUAN A, BERENGUER SÁNCHEZ A.: Análisis comparativo de diferentes técnicas para la corrección de la incontinencia urinaria de stress. Revisión de nuestra experiencia. Actas Urol Esp 1997; 21: 655.

28. IOCCA A, HERSCHORN S.: Pubovaginal sling in the treatment of recurrence female stress incontinence. J Urol 1998; 159 Suppl: abstract 171: 47.

29. PANNEL M, NATALE F, CONTI C, CERVIGNI M.: Vaginal wall sling in the treatment of type III stress urinary incontinence. J Urol 1998; 159 Suppl: abstract 1247: 325.

30. GOUSSE A, SAFIR M, SAFMAN K, RAZ S.: Anterior vaginal wall sling: six years experience with 425 cases. J Urol 1998; 159 Suppl: abstract 1253: 326.

31. RODRIGUES P, ZANETTINI C, HERMANN V, FIGUEIREDO J, LUCENA R, RODRIGUES NETTO N.: Is vaginal sling a good option for intrinsic sphinteric deficiency. J Urol 1998; 159 Suppl: abstract 1245: 324.

32. ZARAGOZA M, DOVER D.: Pubovaginal sling as primary treatment for type 2 stress incontinence: 4 years results. J Urol 1998; 159 Suppl: abstract 1247: 325.

Dr. E. Redondo González

Servicio de Urología. Hospital Univ. de Getafe

Ctra. de Toledo Km 12,500

28905 Getafe (Madrid)

(Trabajo recibido el 3 septiembre de 2002) 